Universidad

BIBLIOTECA

Document downloaded from the institutional repository of the University of Alcala: http://ebuah.uah.es/dspace/

This is a postprint version of the following published document:

Sendra, J.R., Peternell, M. \& Sendra, J. 2018, "Cissoid constructions of augmented rational ruled surfaces", Computer Aided Geometric Design, vol. 60 , pp. 1-9

Available at https://doi.org/10.1016/i.cagd.2017.12.001

(C) 2018 Elsevier

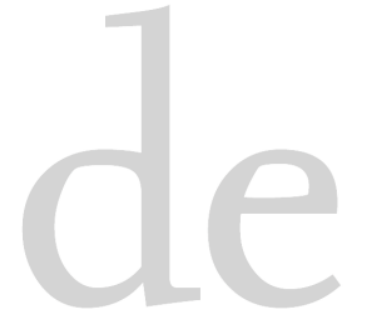

(Article begins on next page)
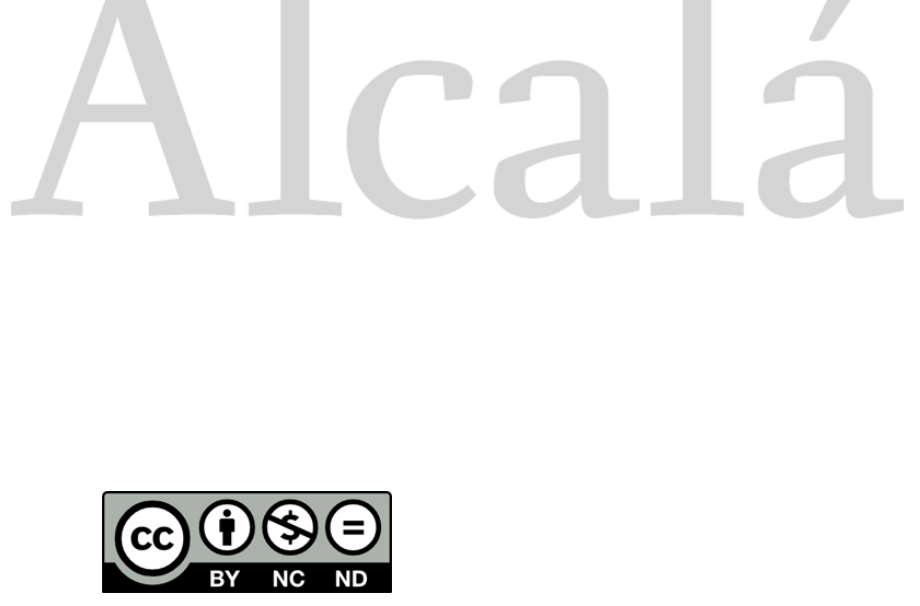

This work is licensed under a

Creative Commons Attribution-NonCommercial-NoDerivatives

4.0 International License. 
The final journal version of this paper appears in [M. Peternell, J.R.Sendra, J. Sendra. Computer Aided Geometric Design 60 (2018) 1-9.] and it is avalialble at https:// doi.org/10.1016/j.cagd.2017.12.001

\title{
Cissoid Constructions of Augmented Rational Ruled Surfaces
}

\author{
J.Rafael Sendra*, Martin Peternell ’ Juana Sendra ${ }^{\ddagger}$
}

\begin{abstract}
The cissoid surface $C=F \diamond G$ of two surfaces $F$ and $G$ with respect to a reference point $O$ is obtained by summing up vectors of points $\mathbf{f} \in F$ and $\mathbf{g} \in G$ being collinear with $O$. In that way the representations of the surfaces $F, G$ and $F \diamond G$ are put into relation by the bundle of lines through $O$.

Typically the construction is non rational. Conditions are studied under which rational surfaces have a cissoid admitting rational parametrizations for generic position of the reference point. In particular, the cissoid of two augmented ruled surfaces or an augmented ruled surface and an augmented quadratically parametrizable surface is rationally parametrizable. Moreover, the construction for two augmented ruled surfaces is even rational if the reference point moves on a rational surface.
\end{abstract}

keyword: Rational surface, ruled surface, quadratically parametrized surface, augmented surface, cissoid, homotopic deformation of surfaces, symbolic computation.

\section{Introduction}

The development of automatic geometric design theory has come accompanied by the study of certain geometric constructions on surfaces, many of them with origins in classical geometry. Probably the most significant and successful one is the offset (parallel surfaces in the

*Rafael.Sendra@uah.es, Research group ASYnACS, Dpto. de Fisica y Matemáticas, Universidad de Alcalá, E-28871 Alcalá de Henares, Madrid, Spain

†peternell@tuwien.ac.at, Institute of Discrete Mathematics and Geometry, Vienna University of Technology, Austria

${ }_{\ddagger}^{\ddagger}$ jsendra@etsist. upm.es, Dpto. Matemática Aplicada a las TIC. Universidad Politcnica de Madrid, Spain 
classical language); e.g. [8]. Others used constructions are pedal, conchoid, or convolution of surfaces (see [7], [11], [12], [16]). In this paper, we deal with a different geometric construction, also with origins in former geometric studies, the cissoid construction. Given two surfaces $F, G$, a reference point $O$, and two numbers $\lambda, \mu \in \mathbb{R}$, the cissoid is the geometric locus of those points $P$ such that

$$
\overline{O P}=\lambda \overline{O X}+\mu \overline{O Y}
$$

for some $X$ in $F$ and some $Y$ in $G$. Note that this formulation is equivalent to consider, as we will do in the rest of the paper, $\overline{O P}=\overline{O X}+\overline{O Y}$ where now $X$ is in the augmented surface $\lambda F$ and $Y$ in the augmented surfaces $\mu G$; a similar reasoning can be done if instead of two numbers $\lambda, \mu$ we consider two deformation functions. This motivates indeed our notion of augmented surface: let $T(u, v)$ be a rational function and $\mathbf{f}(u, v)$ a rational parametrization of a surface $F$, we will call the surface parametrized as $T(u, v) \mathbf{f}(u, v)$ a $T$-augmentation of the surface $F$. When $F$ is ruled or quadratically parametrized we get augmented ruled or augmented quadratically parametrized surfaces, respectively.

For potential applications, one may consider the cissoid as the expression $\lambda \overline{O X}+(1-\lambda) \overline{O Y}$. In this way, when $\lambda$ varies in the real close interval $[0,1]$, the cissoid will describe the homotopic deformation, from the point of view of an observer $O$, of a surface with initial value $F$ and final stage at $G$. This interpretation of the cissoid construction and, consequently, its analysis, could be of help in applications as, for instance, boundary evolution (see [15]) or boundary deformations (see [6], [9]).

In this paper, we will deal with the cissoid of two rational algebraic surfaces and we study the rationality, and actual computation of parametrizations, of the cissoid. Note that a parametrization of the cissoid provides a differentiable map describing the homotopic deformation of one surface into the other. More precisely, our main contribution of the paper is as follows.

Contribution: Let $F, G$ be rational surfaces. We prove that, if $F$ is augmented ruled and $G$ is either augmented ruled or augmented quadratically parametrized, the cissoid of $F$ and $G$ from any reference point $O$ is a rational surface. Furthermore, given rational parametrizations of $F$ and $G$, we provide a rational parametrization of the cissoid. In addition, if the both $F$ and $G$ are augmented ruled, then result is also valid when the reference point $O$ moves on a rationally given surface or space curve.

The paper is structured in three main sections. In Section 2, we introduce the basic preliminaries on cissoids and we show how elimination theory techniques may be applied to find the implicit equations. In Section 3, we characterize the rationality of the cissoid by means of the rationality of an auxiliary variety. This result provides indeed a method to check algorithmically the rationality of the cissoid of two rational surfaces. Finally, in Section 4 we analyze the cissoid of augmented ruled surfaces. 


\section{Preliminaries}

In this section we recall some basic fact required in the paper. More precisely, we recall the cissoid construction, the convolution construction and the polar representation of surfaces.

\subsection{Cissoid construction}

The cissoid construction is a generalization of the conchoid construction, see for instance [1]. Given two surfaces $F$ and $G$, and a fixed reference point $O$, consider a line through $O$ intersecting $F$ at $X$ and $G$ at $Y$. Then the locus of points $P$ with $\overline{O P}=\overline{O X}+\overline{O Y}$ is the cissoid of $F$ and $G$. The construction is analogously defined for planar curves or hypersurfaces.

Cissoid as parametrized surface. Typically the reference point $O$ of the cissoid construction is chosen as origin $O=(0,0,0)$ of a Cartesian coordinate system. Then, the cissoid $F \diamond G$ is represented by vectors $\mathbf{p}=\mathbf{f}+\mathbf{g}$, for linearly dependent position vectors $\mathbf{f} \in F$ and $\mathbf{g} \in G$. Consequently it is defined by

$$
F \diamond G=\{\mathbf{f}+\mathbf{g} \text {, with } \mathbf{f} \in F, \mathbf{g} \in G \text {, and } \mathbf{f} \| \mathbf{g}\} .
$$

The construction is commutative, i.e. $F \diamond G=G \diamond F$. For any point $\mathbf{g} \in G$ there is typically a finite number of corresponding points $\mathbf{f}_{i} \in F$, related to the degree of $F$.

This definition clearly depends on the chosen reference point. Once we have chosen $O$, the definition is invariant with respect to linear or affine maps fixing $O$. Choosing $G$ as the sphere of radius $d$, centered at the origin $O$, the cissoid $F \diamond G$ becomes the conchoid of $F$ with respect to $O$ and distance $d$ (see e.g. [7], [11]).

Cissoid as implicit surface. Since the construction is algebraic, the cissoid of two algebraic surfaces $F$ and $G$ is algebraic. The cissoid can be computed by means of Gröbner bases. In [1], it is shown how to determine the cissoid by means of resultants, but extraneous factors may appear. Let $\mathbf{a}, \mathbf{b}$, and $\mathbf{x}=(x, y, z)$ be vectors $\in \mathbb{R}^{3}$, and consider the polynomials $F(\mathbf{a})$ and $G(\mathbf{b})$ defining the surfaces $F$ and $G$. We do not distinguish between polynomials and surfaces, since it should be clear from the contents, if $F$ or $G$ denotes a polynomial or a surface. To define the cissoid $F \diamond G$ by means of an implicit equation, we Consider the ideal

$$
J=<\mathbf{x}-\mathbf{a}-\mathbf{b}, \mathbf{a} \times \mathbf{b}, F(\mathbf{a}), G(\mathbf{b})>
$$

in the polynomial ring in $\mathbb{C}[\mathbf{a}, \mathbf{b}, \mathbf{x}]$. The first and the second entry in $J, \mathbf{x}-\mathbf{a}-\mathbf{b}$, and $\mathbf{a} \times \mathbf{b}$, correspond directly to equation (1), and this construction is performed for all $\mathbf{a}$ in $F$ and $\mathbf{b} \in G$. This implies that the cissoid $F \diamond G$ is the variety of the ideal $J \cap \mathbb{C}[\mathbf{x}]$. 
On the other hand, if $\mathbf{f}(u, v)$ and $\mathbf{g}(s, t)$ are respective parametrizations of $F$ and $G$, we consider the ideal

$$
J=<\operatorname{numer}(\mathbf{x}-\mathbf{f}-\mathbf{g}), \mathbf{f} \times \mathbf{g}, D(u, v, s, t) W-1>,
$$

in $\mathbb{C}[u, v, s, t, W, \mathbf{x}]$, where $D$ is the least common multiple of all denominators in $\mathbf{f}(u, v)$ and $\mathbf{g}(s, t)$. The cissoid $F \diamond G$ is the variety of the ideal $J \cap \mathbb{C}[\mathbf{x}]$.

\subsection{Convolution construction}

Given two surfaces $F$ and $G$, the convolution $F \star G$ is defined as

$$
F \star G=\left\{\mathbf{f}+\mathbf{g}, \mathbf{f} \in F, \mathbf{g} \in G, \text { with } \mathbf{n}_{f} \| \mathbf{n}_{g}\right\},
$$

where $\mathbf{n}_{f}$ and $\mathbf{n}_{g}$ denote normal vectors of $F$ and $G$ at points $\mathbf{f} \in F$ and $\mathbf{g} \in G$. The definition is affinely invariant by substituting parallel normal vectors by parallel tangent planes at corresponding points, and obviously $F \star G=G \star F$ holds. According to (4), $F \star G$ is the envelope when $G$ is translated with position vectors $\mathbf{f} \in F$, or vice versa. The curve case is illustrated in Fig. 1(a) at hand of the convolution $C$ of a parabola $F$ and an ellipse arc $G$. Parallelity of tangents indicates corresponding points $\mathbf{f} \in F$ and $\mathbf{g} \in G$ such that $\mathbf{c}=\mathbf{f}+\mathbf{g} \in C$.

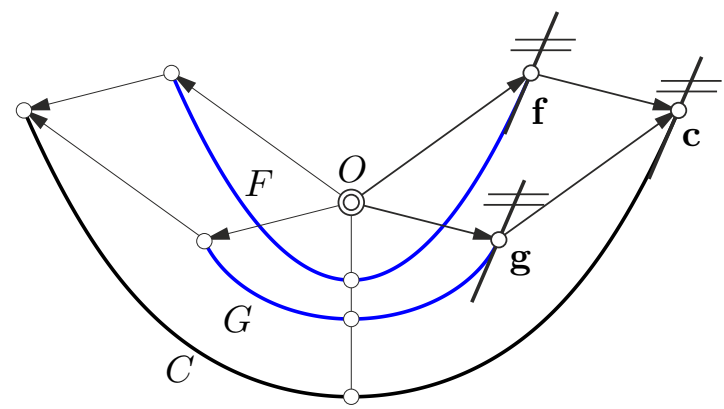

(a) Convolution $C$ of parabola $F$ and ellipse $G$

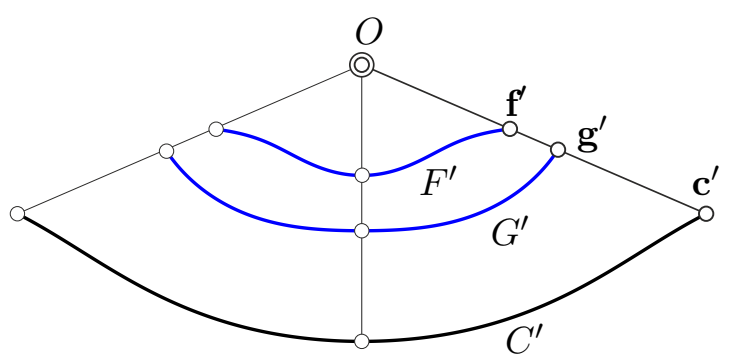

(b) Cissoid $C^{\prime}$ of the respective pedal curves $F^{\prime}$ and $G^{\prime}$ with respect to $O$

Figure 1: Correspondence between the cissoid and the convolution construction

There is a close relation between the convolution and the cissoid construction which is briefly addressed. Consider the surfaces $F$ and $G$ as envelopes of their tangent planes and let $F^{\prime}=\alpha(F), G^{\prime}=\alpha(G)$ and $C^{\prime}=\alpha(C)$ be their respective pedal surfaces with respect to the reference point $O$. Then the cissoid surface of $F^{\prime}$ and $G^{\prime}$ is just $C^{\prime}=F^{\prime} \diamond G^{\prime}$, the pedal surface of $C=F \star G$. Fig. 1(b) illustrated this property for the respective convolution construction in Fig. 1(a). For offset and conchoid surfaces this property is investigated in detail in [11], and analogously it holds for the convolution and cissoid construction. 
The convolution construction is algebraic, and it can be computed with Gröbner bases. For this purpose, let $J$ be the ideal in $\mathbb{C}[\mathbf{x}, \mathbf{a}, \mathbf{b}]$

$$
J=<\mathbf{x}-\mathbf{a}-\mathbf{b}, F(\mathbf{a}), G(\mathbf{b}), \nabla F(\mathbf{a}) \times \nabla G(\mathbf{b})>.
$$

Then, the convolution $F \star G$ is the variety of $J \cap \mathbb{C}[\mathbf{x}]$. In the parametric case, a similar ideal manipulation as in the cissoid case can be performed.

By using the scaled versions $d G$ of $G$ we mean simply the object parameterized by $d \mathbf{g}$ instead of $\mathbf{g}$. In this way families of convolution surfaces and cissoid surfaces, depending on the parameter $d$, are constructed. This is related to offsets for different distances, which are obtained as envelopes of spheres of radius $d$.

\subsection{Polar representation of surfaces}

In order to deal with parametric representations of cissoid surfaces, we use special parametrizations of surfaces. Similar to spherical coordinates, consisting of a radius function and two angles, representing a point in space, we consider parametrizations $\mathbf{f}(u, v)=$ $r(u, v) \mathbf{n}(u, v)$, with $\|\mathbf{n}\|^{2}=1$, where $r(u, v)$ denotes the radius function and $\mathbf{n}(u, v)$ is a parametrization of the unit sphere as reference surface. The parametrization $\mathbf{f}=r \mathbf{n}$ is called a polar representation of the surface $F$.

By choosing the reference point of the cissoid construction as origin $O=(0,0,0)$, we assume that both surfaces $F$ and $G$ are represented by corresponding polar representations (see Def. 1 in [7])

$$
\mathbf{f}(u, v)=r_{1}(u, v) \mathbf{n}(u, v) \text {, and } \mathbf{g}(u, v)=r_{2}(u, v) \mathbf{n}(u, v), \text { with }\|\mathbf{n}\|=1 \text {. }
$$

The correspondence between points in $F$ and $G$ is realized by coinciding parameters $u, v$. Then the cissoid (or a component of it) $C=F \diamond G$ is represented by the polar representation

$$
\mathbf{c}(u, v)=\left(r_{1}+r_{2}\right)(u, v) \mathbf{n}(u, v) .
$$

The use of the unit sphere $S^{2}$ as reference surface for the cissoid as well as the convolution construction is somehow artificial. For the computation of the cissoid $C=F \diamond G$ of two surfaces $F$ and $G$, one might use one surface, say $F$, as reference surface and parameterize the second one over that one. This avoids the dependency on the parametrization of the unit sphere $S^{2}$. The cissoid construction obviously depends essentially on the chosen reference point. Any translation applied to the input surface will change the result. But the construction is of course invariant to affine maps keeping the reference point $O$ unchanged.

Theorem 1 Consider a parametrization $\mathbf{f}(u, v)$ of the surface $F$ and assume that $G$ admits a parametrization of the form $\mathbf{g}(u, v)=m(u, v) \mathbf{f}(u, v)$. In this case, $G$ is parametrized over 
$F$ with respect to $\mathbf{f}(u, v)$. Then the cissoid $C=F \diamond G$ (or a component of it) is represented by

$$
\mathbf{c}(u, v)=(1+m(u, v)) \mathbf{f}(u, v) .
$$

\section{Rationality of the cissoid surface}

We have seen that a particular case of the cissoid is the conchoid construction. In [14] the number of irreducible components is studied in case of the conchoid construction. Although in general the cissoid will be an irreducible variety, reducible components may appear. In the following, we use the notion 'rationality' when one component of the cissoid is rational.

Consider two surfaces $F$ and $G$, given implicitly as zero set of the respective polynomials $F(x, y, z)$ and $G(x, y, z)$. Let $\mathbf{f}(u, v)$ be a proper rational parametrization of $F$. Taking the approach presented in Theorem 1, we try to parametrize $G$ over $F$. Thus, we look for a rational function $m(u, v)$ such that $m(u, v) \mathbf{f}(u, v)$ is a parametrization of $G$. In order to do so we plug $w \mathbf{f}(u, v)$ into $G(x, y, z)$, where $w$ is a new variable. Consequently

$$
H(w, u, v):=\operatorname{numer}(G(w \mathbf{f}(u, v)) \in \mathbb{C}[w, u, v]
$$

is a polynomial in $w$ and $u, v$, and $H(w, u, v)=0$ is called reparametrizing variety. Formally $H$ depends on the chosen parametrization $\mathbf{f}(u, v)$ of $F$. The following Lemma shows that the property that $F$ is parametrizable over $G$ is independent of the chosen parametrization $\mathbf{f}(u, v)$. Theorem 3 characterizes the property of being parametrizable over a surface by means of $H$.

Lemma 2 The property that $G$ is parametrizable over $F$ is independent of the chosen proper parametrization $\mathbf{f}(u, v)$ of $F$.

Proof: Let $\mathbf{f}(u, v)$ and $\overline{\mathbf{f}}(\bar{u}, \bar{v})$ be two different proper parametrizations of $F$. Then there exists an invertible map $\psi(\bar{u}, \bar{v})=(u, v)$ such that $\overline{\mathbf{f}}(\bar{u}, \bar{v})=\mathbf{f}(\psi(\bar{u}, \bar{v}))$. Substituting $(u, v)$ by $\psi(\bar{u}, \bar{v})$ in $\mathbf{g}(u, v)=m(u, v) \mathbf{f}(u, v)$ yields

$$
\begin{array}{r}
\mathbf{g} \circ \psi(\bar{u}, \bar{v})=m \circ \psi(\bar{u}, \bar{v}) \mathbf{f} \circ \psi(\bar{u}, \bar{v}), \\
\mathbf{g}(\bar{u}, \bar{v})=\bar{m}(\bar{u}, \bar{v}) \overline{\mathbf{f}}(\bar{u}, \bar{v}), \text { with } \bar{x}=x \circ \psi .
\end{array}
$$

Thus the property that $m(u, v) \mathbf{f}(u, v)$ parameterizes $G$ is equivalent to that $\bar{m}(\bar{u}, \bar{v}) \overline{\mathbf{f}}(\bar{u}, \bar{v})$ parameterizes $G$.

Theorem 3 Let $D$ be the variety defined by the the least common multiple of the denominators in $\mathbf{f}(u, v)$. The following statements are equivalent 
1. $G$ can be parametrized over $F$ using $\mathbf{f}(u, v)$.

2. The reparametrizing variety $H$ has a rational component, different from any irreducible component of $D$, and this component admits a rational parametrization $\mathbf{q}(s, t)=\left(q_{1}, q_{2}, q_{3}\right)(s, t)$, such that the Jacobian of $\mathbf{g}(s, t)=q_{1}(s, t) \mathbf{f}\left(q_{2}(s, t), q_{3}(s, t)\right)$ has rank 2.

Furthermore, if (2) holds, then $\mathbf{g}(s, t)$ parametrizes $G$.

Proof: Let $d(u, v)$ be a defining polynomial of $D$, and let $\mathbf{g}(u, v):=m(u, v) \mathbf{f}(u, v)$ be a rational parametrization of $G$, which implies $m \neq 0$. Then, $G(\mathbf{g}(u, v))=0$. Thus $H(m(u, v), u, v)=0$, and hence $\mathbf{q}(u, v)=(m(u, v), u, v)$ parametrizes a component of $H$, which is clearly different of $D$. Moreover, $q_{1}(u, v) \mathbf{f}\left(q_{2}(u, v), q_{3}(u, v)\right)=m(u, v) \mathbf{f}(u, v)$, whose Jacobian has rank 2.

Conversely, let $\mathbf{q}(s, t)$ be a rational parametrization of a component of $H$, different of $D$. Then $H(\mathbf{q}(s, t))=0$ and $d\left(q_{2}, q_{3}\right)(s, t) \neq 0$. $H$ can be expressed as $H(w, u, v)=$ $G(w \mathbf{f}(u, v)) d(u, v)^{n}$ for some $n \in \mathbb{N}$. We consider the parametrization

$$
\mathbf{g}(s, t)=q_{1}(s, t) \mathbf{f}\left(q_{2}(s, t), q_{3}(s, t)\right),
$$

which satisfies $G\left(q_{1} \mathbf{f}\left(q_{2}(s, t), q_{3}(s, t)\right)\right)=0$. Since the Jacobian of $\mathbf{g}(s, t)$ has rank two, $\mathbf{g}(s, t)$ is a rational parametrization of $G$ over $F$ using $\mathbf{f}$.

Remark 1 We observe that the condition on the rank of the Jacobian in Theorem 3 cannot be avoided, as the following example shows. Consider the planes $F: x=0$ and $G: y=0$. Clearly, $G$ cannot be parametrized over $F$. However, if $\mathbf{f}(u, v)=(0, u, v)$, then $H(w, u, v)=$ $u$ and $H=0$ is rational. Nevertheless, any parametrization of $H(w, u, v)=0$ is of the form $(s, 0, t)$. But obviously the Jacobian of the parametrization $s \mathbf{f}(0, t)=s(0,0, t)$ has rank 1.

As a consequence of equation (8) and of Theorem 3, we have the following criterium for detecting rational cissoids.

\section{Corollary 4 [Criterium of rationality]}

Let $\mathbf{f}(u, v)$ be proper parametrization of $F$ and the assumptions on $D$ according to Theorem 3. If $H$ has a rational component $\mathbf{q}(s, t)=\left(q_{1}, q_{2}, q_{3}\right)(s, t)$ different from any irreducible component of $D$ and if the Jacobian of $\mathbf{g}=q_{1} \mathbf{f}\left(q_{2}, q_{3}\right)$ has rank 2 , then the cissoid $C=F \diamond G$ has a component parametrized by

$$
\mathbf{c}(s, t)=\mathbf{f}(s, t)+\mathbf{g}(s, t)=\left(1+q_{1}(s, t)\right) \mathbf{f}\left(q_{2}(s, t), q_{3}(s, t)\right) .
$$


Example 5 Let $F$ be the quadric defined by $F(x, y, z)=-x z+y+1$ and $G$ be the cubic surface defined by $G(x, y, z)=x^{2} y-z+1 . F$ is a hyperbolic paraboloid, thus a ruled quadric, and $G$ is a ruled cubic surface, equivalent to the Whitney umbrella $z y^{2}=x^{2}$, up to a projective transformation $((x, y, z) \mapsto(x / y, 1 / y, z / y)$ composed with a translation). Using the ideal introduced in equation (2), the cissoid $C=F \diamond G$ is a 10-degree surface defined by

$x^{5} y^{2} z^{3}-3 x^{4} y^{3} z^{2}-3 x^{4} y^{2} z^{2}+3 x^{3} y^{4} z-2 x^{3} y z^{4}+6 x^{3} y^{3} z+2 x^{3} y z^{3}-x^{2} y^{5}+4 x^{2} y^{2} z^{3}+$ $3 x^{3} y^{2} z-3 x^{2} y^{4}-3 x^{2} z^{2} y^{2}-3 x y^{3} z^{2}+x z^{5}-3 x^{2} y^{3}+6 x^{2} y z^{2}+3 x y^{3} z-2 x z^{4}+z y^{4}-z^{4} y-$ $x^{2} y^{2}-3 x y^{2} z+2 x y z^{2}+x z^{3}-y^{4}+y^{3} z+y z^{3}-z^{4}$.

$F$ is parametrizable by $\mathbf{f}(u, v)=(u, u v-1, v)$, and consequently the reparametrizing variety $H$ is defined by

$$
H(w, u, v)=w^{3} u^{3} v-w^{3} u^{2}-w v+1 .
$$

Since $H$ is linear in $v$, it is obviously parametrized as

$$
\mathbf{q}(u, w)=\left(w, u, \frac{u^{2} w^{3}-1}{w\left(u^{3} w^{2}-1\right)}\right)=(w, u, v(u, w)) .
$$
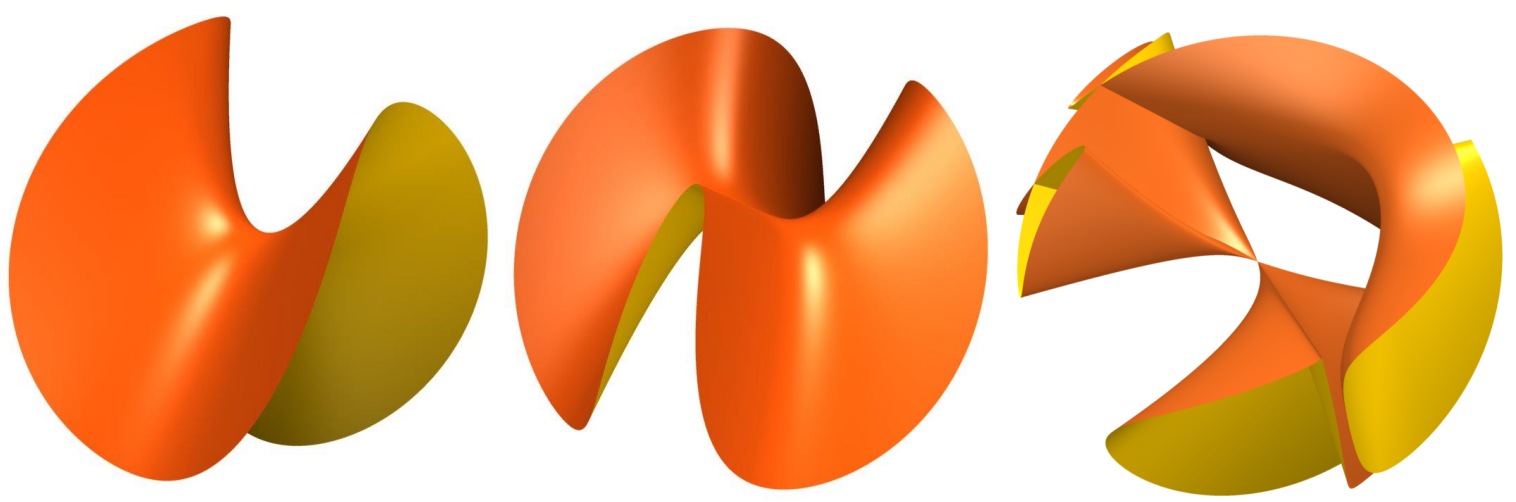

Figure 2: $F$ (left), $G$ (center), $F \diamond G$ (right) in Example 5

By Corollary 4, the cissoid surface $C=F \diamond G$ is rational and can be parametrized as

$$
\begin{aligned}
\mathbf{c}(u, w) & =\left(1+q_{1}(u, w)\right) \mathbf{f}\left(q_{2}(u, w), q_{3}(u, w)\right), \\
& =(1+w)(u, u v(u, w)-1, v(u, w)),
\end{aligned}
$$

and the Jacobian of $\mathbf{g}(u, w)=q_{1}(u, w) \mathbf{f}\left(q_{2}(u, w), q_{3}(u, w)\right)$ has rank 2 .

Corollary 6 Let $F$ be a rational surface and let $G$ be a plane, where $O \notin G$. Then, the cissoid $P=F \diamond G$ is rational. 
Proof: Let $\mathbf{f}(u, v)$ and $G=a_{1} x+a_{2} y+a_{3} z+a_{4}=\mathbf{a} \cdot \mathbf{x}+a_{4} . O \notin G$ implies $a_{4} \neq 0$. To construct a rational parametrization $\mathbf{g}(u, v)=w(u, v) \mathbf{f}(u, v)$ of $G$, the lines $w \mathbf{f}$ are intersected with $G$. The unique intersection point leads to

$$
w(u, v)=-a_{4} /(\mathbf{a} \cdot \mathbf{f}(u, v)) .
$$

This agrees with the fact that the reparametrizing variety $H$ is the numerator of $w(\mathbf{a}$. $\mathbf{f}(u, v))+a_{4}$ and consequently $H$ is parametrized by $\mathbf{q}(u, v)=\left(-a_{4} /(\mathbf{a} \cdot \mathbf{f}), u, v\right)$. The plane $G$ and the cissoid $C=F \diamond G$ are parametrized by

$$
\mathbf{g}(u, v)=\frac{-a_{4}}{\mathbf{a} \cdot \mathbf{f}(u, v)} \mathbf{f}(u, v) \text {, and } \mathbf{c}(u, v)=\mathbf{f}(u, v)+\mathbf{g}(u, v)=\frac{\mathbf{a} \cdot \mathbf{f}(u, v)-a_{4}}{\mathbf{a} \cdot \mathbf{f}(u, v)} \mathbf{f}(u, v) .
$$

Depending on $\mathbf{f}$, the parametrization $\mathbf{g}$ of $G$ is not necessarily proper.

\section{The cissoid surface of an augmented ruled surface}

Let us start this section introducing the notion of augmented ruled surface. We say that a rational surface is an augmented ruled surface, if it admits a parametrization of the form

$$
T(u, v)(\mathbf{a}(u)+v \mathbf{b}(u))
$$

where $T$ is a rational function in $u, v$.

We study the cissoid $C=F \diamond G$ of two rational surfaces $F$ and $G$, where one, say $F$, is an augmented ruled surface. At first we describe the general strategy for the analysis of the rationality of the cissoid. Later this is applied to some special cases.

Let $F$ and $G$ be represented by the respective parametrizations $\mathbf{f}(u, v)=T(u, v) \widetilde{\mathbf{f}}(u, v)$ where $\widetilde{\mathbf{f}}(u, v)=\mathbf{a}(u)+v \mathbf{b}(u)$, and $\mathbf{g}(s, t)$. Consider the family of planes $\alpha(u): \mathbf{x} \cdot(\mathbf{a}(u) \times$ $\mathbf{b}(u))=0$ passing through $O$ and the generating lines of the ruled surface $\widetilde{F}$ defined by $\widetilde{\mathbf{f}}$. Intersecting $G$ with the planes $\alpha(u)$ yields a family of curves $a(u)=G \cap \alpha(u)$, see Fig. 3 . Inserting the parametrization $\mathbf{g}(s, t)$ into $\alpha(u)$ results in an implicit representation of these curves $a(u)$

$$
A(s, t ; u)=\mathbf{g}(s, t) \cdot(\mathbf{a} \times \mathbf{b})(u)=0 .
$$

Let us assume that $A(s, t ; u)=0$ defines a family of rational curves in the st-plane, with family parameter $u$. Then there exists a rational parametrization

$$
\varphi(u, w)=(s(u, w), t(u, w)), \text { with } A(s(u, w), t(u, w) ; u)=0 .
$$

Substituting $\varphi(u, w)$ into $\mathbf{g}(s, t)$ yields $\mathbf{g}(s(u, w), t(u, w))=: \mathbf{g}(u, w)$, such that the $w$-lines of $\mathbf{g}(u, w)$ are the planar curves $a(u)=G \cap \alpha(u)$. Consequently $\operatorname{det}(\mathbf{g}(u, w), \mathbf{a}(u), \mathbf{b}(u))=0$ holds. 
The system of equations

$$
\lambda \mathbf{g}(u, w)=\mathbf{a}(u)+v \mathbf{b}(u)=\widetilde{\mathbf{f}}(u, v)
$$

is linear in $\lambda$ and $v$, and has a rational solution $\lambda(u, w), v(u, w)$. Substituting $v(u, w)$ in $\mathbf{f}(u, v)$ yields $\mathbf{f}(u, v(u, w))=: \mathbf{f}(u, w)$. This implies a rational parametrization $\mathbf{f}(u, w)=$ $T(u, w) \lambda(u, w) \mathbf{g}(u, w)$ of $F$ with the property that $\mathbf{f}(u, w)$ and $\mathbf{g}(u, w)$ are linearly dependent. Finally the cissoid $C=F \diamond G$ is rationally parametrized by

$$
\begin{aligned}
\mathbf{c}(u, w) & =\mathbf{f}(u, v(u, w))+\mathbf{g}(u, w) \\
& =(1+T(u, v(u, w)) \lambda(u, w) \mathbf{g}(s(u, w), t(u, w)) .
\end{aligned}
$$

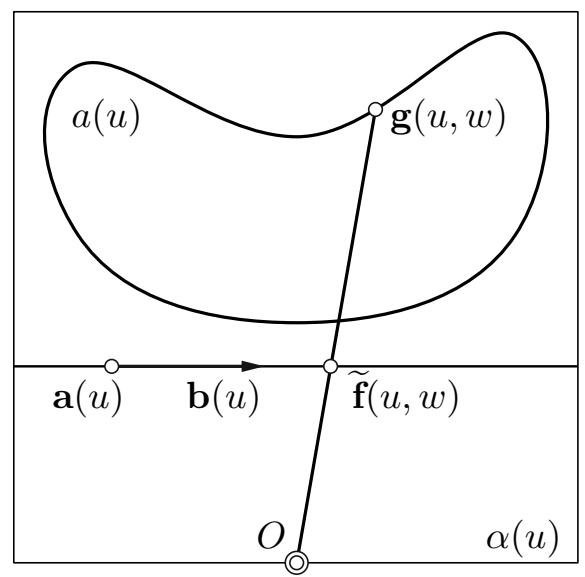

Figure 3: Cissoid construction of augmented ruled surfaces

Theorem 7 Let $F$ and $G$ be two rational surfaces where $F$ is augmented ruled. The respective parametrizations are $\mathbf{f}(u, v)=T(u, v)(\mathbf{a}(u)+v \mathbf{b}(u))$ and $\mathbf{g}(s, t)$. If the generic planar intersection curve $a(u)=\alpha(u) \cap G$, with a plane $\alpha(u): \mathbf{x} \cdot(\mathbf{a}(u) \times \mathbf{b}(u))=0$, is rational, then the cissoid surface $C=F \diamond G$, with respect to any reference point $O \in \mathbb{R}^{3}$, is rationally parametrized by (14).

According to this statement, a sufficient condition for a rational construction of the cissoid surface is that the generic planar intersections of $G$ are rational. The next sections describe these cases in detail. For the case of ruled surfaces, i.e. $T$ being a rational function depending only on $u$, it is known that there are only two possibilities, and $G$ has to be either a ruled surface or it is a quadratically parametrizable surface, see for instance [3], Vol 4, page 55. The quadratically parametrizable surfaces are projections of a Veronese surface of degree four, and often denoted as Steiner surfaces. The most famous representative is Steiner's roman surface, see for instance [4], [5]. In the next section, we analyze the cases where $G$ is either an augmented ruled surface or an augmented quadratically parametrized surface. 


\subsection{Two augmented ruled surfaces}

Let $F$ and $G$ be two augmented ruled surfaces, represented by the respective parametrizations $\mathbf{f}(u, v)=T_{1}(u, v) \widetilde{\mathbf{f}}(u, v)$ and $\mathbf{g}(s, t)=T_{2}(s, t) \widetilde{\mathbf{g}}(s, t)$ where $\widetilde{\mathbf{f}}(u, v)=\mathbf{a}(u)+v \mathbf{b}(u)$ and $\widetilde{\mathbf{g}}(s, t)=\mathbf{c}(s)+t \mathbf{d}(s)$. The intersection curves $a(u)=G \cap \alpha(u)$, with planes $\alpha(u)$ : $\mathbf{x} \cdot(\mathbf{a}(u) \times \mathbf{b}(u))=0$ through $O$ and the generating lines of $\widetilde{\mathbf{f}}$ are determined by

$$
A(s, t ; u)=(\mathbf{c}(s)+t \mathbf{d}(s)) \cdot(\mathbf{a}(u) \times \mathbf{b}(u))=0 .
$$

This equation can directly be solved for $t$ by,

$$
t(s, u)=-\frac{\operatorname{det}(\mathbf{a}, \mathbf{b}, \mathbf{c})(s, u)}{\operatorname{det}(\mathbf{a}, \mathbf{b}, \mathbf{d})(s, u)} .
$$

Substituting $t(s, u)$ into $\widetilde{\mathbf{g}}(s, t)$ yields a reparametrization $\widetilde{\mathbf{g}}(s, u)$ with the property that $\operatorname{det}(\widetilde{\mathbf{g}}(s, u), \mathbf{a}(u), \mathbf{b}(u))=0$. Thus the system $\lambda \widetilde{\mathbf{g}}(s, u)=\mathbf{a}(u)+v \mathbf{b}(u)$ has a rational solution $\lambda(s, u), v(s, u)$. Finally the cissoid $C=F \diamond G$ is parametrized by

$$
\begin{aligned}
\mathbf{c}(s, u) & =\mathbf{f}(u, v(s, u))+\mathbf{g}(s, t(s, u)) \\
& =\left(\lambda(s, u) T_{1}(u, v(s, u))+T_{2}(s, t(s, u))\right) \widetilde{\mathbf{g}}(s, t(s, u)) .
\end{aligned}
$$

This result can be generalized in the way that we are allowed to replace the fixed chosen reference point $O$ by a reference point, whose position depends rationally on parameters, see Section 4.3.

Corollary 8 The cissoid surface $F \diamond G$ of two augmented ruled surfaces $F$ and $G$ is a rational surface, independently of the chosen reference point.

Example 9 We consider the ruled surfaces $F$ and $G$ given parametrically by

$$
\mathbf{f}(u, v)=\left(v+u+2, u^{2}+v+1, u^{3}+u v+1\right), \mathbf{g}(s, t)=\left(t+1, s+1, s t+s^{2}+1\right)
$$

The respective implicit equations are

$$
\begin{array}{r}
F=-x y^{2}+y^{3}+2 y x-y^{2}+y z-z^{2}-x-2 y+z+1=0, \\
G=y x+y^{2}-x-3 y-z+3=0 .
\end{array}
$$

According to equation (15), one obtains

$$
t(u, s)=-\frac{s^{2} u^{2}-s u^{3}-s^{2} u+s u^{2}-u^{3}-s^{2}+2 s u+2 u^{2}-s-1}{s u^{2}-s u-s-u+1} .
$$



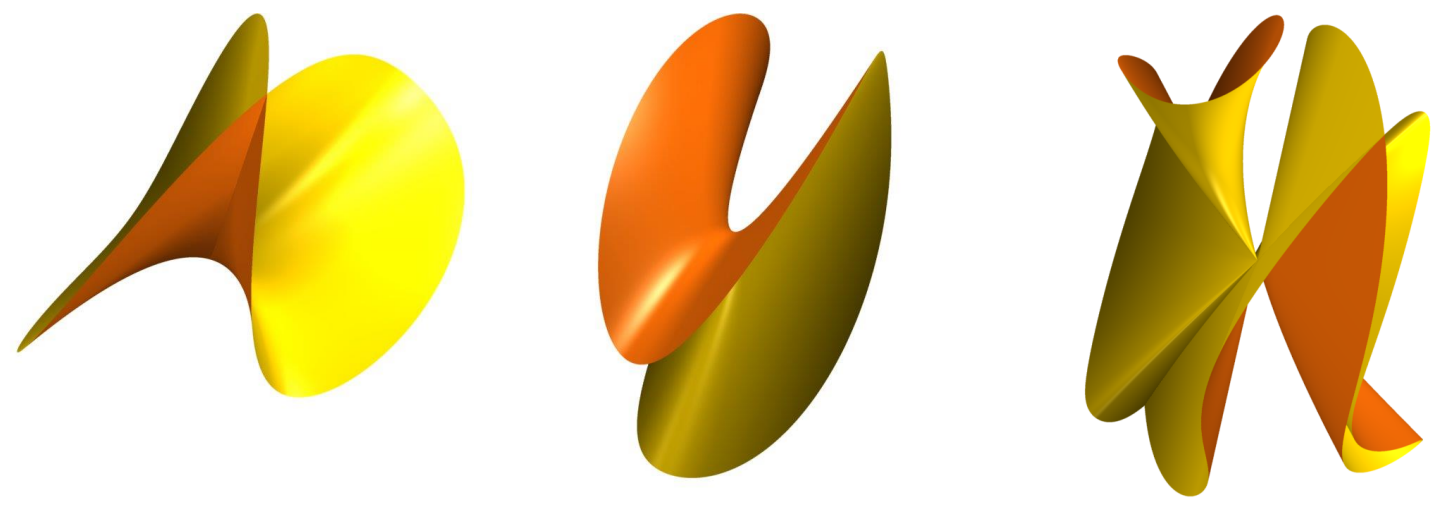

Figure 4: $F$ (left), $G$ (center), $F \diamond G$ (right) in Example 9

solving the linear system corresponding to $\lambda(u, s) \mathbf{g}(s, u)=\mathbf{f}(u, v(u, s))$ yields

$$
\lambda(u, s)=\frac{s u^{2}-s u-s-u+1}{2 s^{2}-s u-u+1} .
$$

Finally we compute the parametrization of the cissoid, which is of degree 11. Since the parametrization has 144 terms, we omitted displaying it.

\subsection{Augmented ruled surface and augmented quadratically parametriz- able surface}

A surface parametrized by

$$
\frac{1}{q_{0}(s, t)}\left(q_{1}, q_{2}, q_{3}\right)(s, t),
$$

where $q_{i}(s, t)$ are polynomials of degree at most two, and $\operatorname{gcd}\left(q_{0}, q_{1}, q_{2}, q_{3}\right)=1$ is called quadratically parametrizable, see for instance [4] or [5]. We defined an augmented quadratically parametrizable surface, as a surface parametrized by

$$
T(s, t) \mathbf{g}(s, t)
$$

where $T$ is a rational function and $\mathbf{g}(s, t)$ a quadratically parametrized surface.

Let $F$ be an augmented ruled surface given by $\mathbf{f}(u, v)=T_{1}(u, v) \widetilde{\mathbf{f}}(u, v)$ where $\widetilde{\mathbf{f}}(u, v)=$ $\mathbf{a}(u)+v \mathbf{b}(u)$, and let $G$ be parametrized by $\mathbf{g}(s, t)=T_{2}(s, t) \widetilde{\mathbf{g}}(s, t)$ with

$$
\widetilde{\mathbf{g}}(s, t)=\frac{1}{q_{0}(s, t)}\left(q_{1}, q_{2}, q_{3}\right)(s, t),
$$

where $q_{i}(s, t)$ are polynomials of degree at most two, and $\operatorname{gcd}\left(q_{0}, q_{1}, q_{2}, q_{3}\right)=1$. We denote by $\widetilde{F}$ and $\widetilde{G}$ the surfaces parametrized by $\widetilde{\mathbf{f}}$ and $\widetilde{\mathbf{g}}$ respectively. 
The intersection curve $a(u)=\widetilde{G} \cap \alpha(u)$ of $\widetilde{G}$ with a plane $\alpha(u): \mathbf{x} \cdot(\mathbf{a} \times \mathbf{b})(u)=0$ through $O$ and the lines of $\widetilde{F}$ is determined by

$$
\widetilde{\mathbf{g}}(s, t) \cdot(\mathbf{a} \times \mathbf{b})(u)=0 .
$$

For any fixed value $u=u^{\star}$ this equation defines a curve of degree two in the st-plane. By applying a coordinate transformation depending on $u$, we obtain the normal form

$$
A(s, t ; u)=r_{0}(u)+r_{1}(u) s^{2}+r_{2}(u) t^{2}=0 .
$$

In case that $A(s, t ; u)=0$ contains real points for almost all values of $u$, there exists a real rational parametrization $\tilde{\varphi}(u, w)=(\tilde{s}(u, w), \tilde{t}(u, w))$ satisfying (20) identically for all $u$. These rational parametrizations can be computed explicitly by solving a linear system and an additional quadratic equation, see [13].

In case that $A(s, t ; u)=0$ contains real points only for $u \in\left[a_{1}, a_{2}\right] \in \mathbb{R}$, one would have to reparametrize the family $A(s, t ; u)=0$ by a quadratic reparametrization $u=$ $\left(a_{2} x^{2}+a_{1}\right) /\left(x^{2}+1\right)$, in order to obtain a real curve $A(s, t ; x)=0$ for all $x \in \mathbb{R}$.

Inverting the coordinate transformation one obtains a rational parametrization $\varphi(u, w)=$ $(s(u, w), t(u, w))$ of (19). Substituting $\varphi(u, w)=(s, t)(u, w)$ into $\widetilde{\mathbf{g}}(s, t)$ yields a oneparameter family of rational curves $\widetilde{\mathbf{g}}(s(u, w), t(u, w))$ in $\widetilde{G}$. These curves are typically of degree four since any generic planar intersection of a quadratically parametrizable surface has this property.

Based on the reparametrization $\varphi(u, w)$ of $\widetilde{G}$ we solve $\lambda \widetilde{\mathbf{g}}(\varphi(u, w))=\mathbf{a}(u)+v \mathbf{b}(u)$ for $\lambda$ and $v$. A rational solution $(\lambda(u, w), v(u, w))$ implies that the cissoid surface $C=F \diamond G$ of the augmented ruled surface $F$ and the augmented quadratically parametrizable surface $G$ admits the probably improper rational parametrization

$$
\begin{aligned}
\mathbf{c}(u, w) & =\mathbf{f}(u, v(u, w))+\mathbf{g}(u, w)= \\
& =\left(\lambda(u, w) T_{1}(\varphi(u, w))+T_{2}(u, v(u, w))\right) \widetilde{\mathbf{g}}(\varphi(u, w)) .
\end{aligned}
$$

The improperness may be caused by an improper real rational parametrization $\varphi(u, w)$ of $A(s, t ; u)=0$.

Corollary 10 The cissoid surface $C=F \diamond G$ of an augmented rational ruled surface $F$ and a irreducible quadric $G$ is a rational surface, independently of the chosen reference point.

Corollary 11 The cissoid surface $F \diamond G$ of an augmented rational ruled surface $F$ and an augmented quadratically parametrizable surface $G$ is a rational surface, independently of the chosen reference point. 
Example 12 We illustrate the cissoid construction for a ruled surface and a quadric. Consider the ruled surface $F: x^{3}-x y z-x^{2}+y^{2}=0$ that is parametrized by

$$
\mathbf{f}(u, v)=\left(v u+1, u^{2} v+u, v+u\right) .
$$

Let the ellipsoid $G: 4 x^{2}+4 y^{2}+z^{2}-4=0$ be parametrized by

$$
\mathbf{g}(s, t)=\left(2 \frac{s}{s^{2}+t^{2}+1}, 2 \frac{t}{s^{2}+t^{2}+1}, 2 \frac{s^{2}+t^{2}-1}{s^{2}+t^{2}+1}\right) .
$$

We get $A(s, t ; u)=-s u+t=0$ and thus $t=u s$. The linear system $\lambda \mathbf{g}(u, s)=\mathbf{f}(u, v)$ results in

$$
\lambda(u, s)=\frac{\left(u^{2}-1\right)\left(u^{2} s^{2}+s^{2}+1\right)}{2\left(s u^{2}+u+s\right)(u s-1)} .
$$

Finally, we get that a parametrization of $F \diamond G$ is given by $\mathbf{p}(u, s)=\left(\frac{p_{1}}{p}, \frac{p_{2}}{p}, \frac{p_{3}}{p}\right)$, where

$$
\begin{aligned}
& p_{1}(u, s)=\left(u^{4} s^{2}+2 u^{3} s^{2}+2 u s^{2}+u^{2}-s^{2}-2 u-2 s-1\right) s \\
& p_{2}(u, s)=\left(u^{4} s^{2}+2 u^{3} s^{2}+2 u s^{2}+u^{2}-s^{2}-2 u-2 s-1\right) u s \\
& p_{3}(u, s)=\left(u^{4} s^{2}+2 u^{3} s^{2}+2 u s^{2}+u^{2}-s^{2}-2 u-2 s-1\right)\left(u^{2} s^{2}+s^{2}-1\right) \\
& p(u, s)=\left(u^{2} s+u+s\right)(u s-1)\left(u^{2} s^{2}+s^{2}+1\right)
\end{aligned}
$$

We observe that in this case the cissoid has degree 8 and its defining polynomial is

$$
\begin{array}{r}
C=4 x^{8}+z^{2} x^{4}-2 z^{2} x^{5}+z^{2} x^{6}-8 x^{7}+8 x^{5} y z+8 x^{4} y z-2 x^{4} y z^{3}+2 y x^{3} z^{3} \\
-8 y z x^{6}+x^{2} y^{2} z^{4}-6 y^{2} z^{2} x^{2}+4 y^{2} x^{6}+4 y^{2} z^{2} x^{4}+2 x^{3} y^{2} z^{2}-4 y^{2} x^{4} \\
-2 x y^{3} z^{3}-8 x^{4} y^{3} z+4 y^{4} z^{2} x^{2}-4 y^{4} x^{2}+8 x^{3} y^{4}+y^{4} z^{2}-8 x y^{5} z+4 y^{6} .
\end{array}
$$

Example 13 Finally we illustrate the method for a ruled surface $F$ and a quadratically parametrizable surface $G$. The surfaces have the respective parametrizations

$$
\mathbf{f}(u, v)=\left(u, v, u^{2}+1\right) \text {, and } \mathbf{g}(s, t)=\left(s, s^{2}+t, 1+t^{2}\right) .
$$

$F$ is a parabolic cylinder with y-parallel lines and $G$ is obtained by translating the parabolas $\left(s, s^{2}, 1\right)$ and $\left(0, t, t^{2}\right)$ along each other. The respective defining polynomials are

$$
F=z-x^{2}-1, \text { and } G=z-\left(y-x^{2}\right)^{2}-1 .
$$

Inserting $\mathbf{g}$ into the equation $\alpha(u):\left(1+u^{2}\right) x-u z=0$ of the planes through the lines of $G$ yields $A(s, t ; u)=-\left(1+u^{2}\right) s+u\left(1+t^{2}\right)$, which is simply solved by $s(t, u)=u\left(1+t^{2}\right) /\left(1+u^{2}\right)$. Substituting this expression in $\mathbf{g}(s, t)$, and solving the linear system $\lambda \mathbf{g}(t, u)=\mathbf{f}(u, v)$ gives $\lambda(t, u)=\left(u^{2}+1\right) /\left(t^{2}+1\right)$ and

$$
v(t, u)=\left(t u^{4}+2 t u^{2}+t+u^{2} t^{4}+2 u^{2} t^{2}+u^{2}\right) /\left(u^{2} t^{2}+u^{2}+t^{2}+1\right) .
$$

Finally, the cissoid $F \diamond G$, of implicit degree 12, is parametrized by

$$
\begin{aligned}
\mathbf{c}(t, u) & =\mathbf{f}(u, v(t, u))+\mathbf{g}(t, u)=(1+\lambda(t, u)) \mathbf{g}(t, u) \\
& =\left(\frac{u\left(u^{2}+2+t^{2}\right)}{u^{2}+1}, \frac{\left(u^{2}+2+t^{2}\right)\left(t u^{4}+2 t u^{2}+t+u^{2} t^{4}+2 u^{2} t^{2}+u^{2}\right)}{\left(t^{2}+1\right)\left(u^{2}+1\right)^{2}}, u^{2}+2+t^{2}\right) .
\end{aligned}
$$




\subsection{Two augmented ruled surfaces and moving reference point}

Let $F$ and $G$ be two augmented ruled surfaces, represented by the respective parametrizations $\mathbf{f}(u, v)=T_{1}(u, v) \widetilde{\mathbf{f}}(u, v)$ and $\mathbf{g}(s, t)=T_{2}(s, t) \widetilde{\mathbf{g}}(s, t)$, where $\widetilde{\mathbf{f}}(u, v)=\mathbf{a}(u)+v \mathbf{b}(u)$ and

$\widetilde{\mathbf{g}}(s, t)=\mathbf{c}(s)+t \mathbf{d}(s)$. Let $\widetilde{F}$ and $\widetilde{G}$ the ruled surfaces given by $\widetilde{\mathbf{f}}$ and $\widetilde{\mathbf{g}}$, respectively. Let the reference point $O$ be given by the position vector $\mathbf{r}(\mathbf{p})$, depending on parameters $\mathbf{p}$ in a rational way.

The planes through $O$ and the lines of $\widetilde{F}$ are now given by $\alpha(u, \mathbf{p}):(\mathbf{x}-\mathbf{r}(\mathbf{p})) \cdot \mathbf{n}(u)=0$, with $\mathbf{n}=\mathbf{a} \times \mathbf{b}$. The intersection curves $a(u, \mathbf{p})=\widetilde{G} \cap \alpha(u, \mathbf{p})$ are determined by

$$
A(s, t ; u, \mathbf{p})=(\mathbf{c}(s)+t \mathbf{d}(s)-\mathbf{r}(\mathbf{p})) \cdot \mathbf{n}(u)=0 .
$$

This equation can directly be solved for $t$ by,

$$
t(s, u, \mathbf{p})=\frac{(\mathbf{r}(\mathbf{p})-\mathbf{c}(s)) \cdot \mathbf{n}(u)}{\mathbf{d}(s) \cdot \mathbf{n}(u)} .
$$

Substituting $t(s, u, \mathbf{p})$ into $\widetilde{\mathbf{g}}(s, t)$ yields a reparametrization $\widetilde{\mathbf{g}}(s, u, \mathbf{p})$ with the property that $\operatorname{det}(\widetilde{\mathbf{g}}(s, u, \mathbf{p}), \widetilde{\mathbf{f}}(u, v), \mathbf{r}(\mathbf{p}))=0$.

Thus the system $(1-\lambda) \mathbf{r}(\mathbf{p})+\lambda \widetilde{\mathbf{g}}(s, u, \mathbf{p})=\mathbf{a}(u)+v \mathbf{b}(u)$ has a rational solution $\lambda(s, u, \mathbf{p})$, $v(s, u, \mathbf{p})$. Finally the family of cissoids $C(\mathbf{p})=F \diamond G$ with respect to the moving reference point $O=\mathbf{r}(\mathbf{p})$ is parametrized by

$$
\mathbf{c}(s, u, \mathbf{p})=\mathbf{g}(s, t(s, u, \mathbf{p}))+T_{1}(u, v(s, u, \mathbf{p})) \lambda(s, u, \mathbf{p})(\widetilde{\mathbf{g}}(s, u, \mathbf{p})-\mathbf{r}(\mathbf{p})) .
$$

Corollary 14 The family of cissoid surfaces $C(\mathbf{p})=F \diamond G$ of two augmented rational ruled surfaces $F$ and $G$ with respect to the rationally moving reference point $O=\mathbf{r}(\mathbf{p})$, consists of rational surfaces, with rational dependency on the parameters $\mathbf{p}$.

\section{Acknowledgments}

This work has been partially funded by Ministerio de Economia y Competitividad, and by the European Regional Development Fund (ERDF), under the Project MTM2014-54141-P. Last two authors belongs to the Research Group ASYNACS (Ref. CCEE2011/R34). Part of this work was developed while M. Peternell was visiting J.R. Sendra at the Universidad de Alcalá in the frame of the project Giner de los Rios. 


\section{References}

[1] Albano, A. and Roggero, M., 2010. Conchoidal transform of two plane curves, Applicable Algebra in Engineering, Communication and Computing, Vol.21, No.4, 309-328.

[2] Arrondo, E., Sendra, J., Sendra, J.R., 1997. Parametric Generalized Offsets to Hypersurfaces. Journal of Symbolic Computation 23, 267-285.

[3] Baker, H.F., 1925. Principles of geometry, Vol 4, Higher Geometry, Cambridge Univ. Press, New York.

[4] Coffman, A., Schwartz, A. J. and Stanton, C. M., 1996. The algebra and geometry of Steiner and other quadratically parametrizable surfaces, Computer Aided Geometric Design 13, 257-286.

[5] Degen, W. L. F., 1996. The types of triangular Bezier surfaces, in: G.Mullineux, ed., The Mathematics of Surfaces VI , 153170, Oxford University Press.

[6] K. Fujimura, E. Kuo. Shape Reconstruction from Contours Using Isotopic Deformation. Graphical Models and Image Processing, Volume 61, Issue 3, 1999, Pages $127-147$.

[7] Gruber D., Peternell M., 2013. Conchoid surfaces of quadrics. Journal of Symbolic Computation 59, 36-53.

[8] Hoschek, J., Lasser, D.: Fundamentals of Computer Aided Geometric Design. A.K. Peters, Ltd. Natick, MA, USA (1993)

[9] L. Jaillet, T. Siméon. Path Deformation Roadmaps. In Algorithmic Foundation of Robotics VII: Selected Contributions of the 7th International Workshop on the Algorithmic Foundations of Robotics. Springer Verlag, 2008, pp. 19-34.

[10] Lávička, M., Bastl, B., 2007. Rational hypersurfaces with rational convolutions. Computer Aided Geometric Design 24 (7), 410-426.

[11] Peternell, M., Gotthart, L., Sendra, J. and Sendra, J.R., 2015. Offsets, conchoids and pedal surfaces. J. Geom. 106, 321-339.

[12] H. Pottmann, W. Lü, B. Ravani. Rational Ruled Surfaces and Their Offsets. Graphical Models and Image Processing, Volume 58, Issue 6, November 1996, Pages 544-552

[13] Schicho J., 2000. Proper Parametrization of Real Tubular Surfaces, J. Symbolic Computation 30, 583-593.

[14] Sendra, J., Sendra, J.R., 2008. An algebraic analysis of conchoids to algebraic curves. Appl. Algebra Eng. Commun. Comput. 19, 413-428 (2008). 
[15] Siddiqi K., Pizer S. (Eds.) Medial Representations Mathematics, Algorithms and Applications. Springer, 2008.

[16] Vršsek J., Lávička M., 2010. On convolutions of algebraic curves. Journal of Symbolic Computation 45, 657-676 